\title{
A New Animal Diet Based On Human Western Diet Is A Robust Diet-Induced Obesity Model: Comparison To High-Fat And Cafeteria Diets In Term Of Metabolic And Gut Microbiota Disruption
}

Calixto Bortolin, Rafael; Rodrigues Vargas, Amanda; Gasparotto, Juciano; Rodrigues Chaves, Paloma; Schnorr, Carlos Eduardo; Da Boit Martinello, Katia; Kleber Silveira, Alexandre; Rabelo, Thallita Kelly;Gelain, D. P; Moreira Fonseca, Jose Claudio.

\section{Abstract}

Background/Objectives:Obesity is a metabolic disorder that predisposes patients to numerous diseases and has become a major global public-health concern. Animal models of diet-induced obesity (DIO) are frequently used to study obesity, but which DIO model most accurately reflects the pathology of human obesity remains unclear. In this study, we designed a diet based on the human Western diet (WD) and compared it with the cafeteria diet (CAF) and high-fat diet (HFD) in order to evaluate which diet most closely mirrors human obesity.Methods:Wistar rats were fed four different diets (WD, CAF, HFD and a low-fat diet) for 18 weeks. Metabolic parameters and gut microbiota changes were then characterized.Results:Rats fed the four different diets exhibited completely different phenotypes, highlighting the importance of diet selection. This study also revealed that WD most effectively induced obesity and obesity-related disorders, and thus proved to be a robust model of human obesity. Moreover, WD-fed rats developed obesity and obesityrelated comorbidities independent of major alterations in gut microbiota composition (dysbiosis), whereas CAF-fed rats developed the greatest dysbiosis independent of obesity. We also characterized gut microbiota after feeding on these four different diets and identified five genera that might be involved in the pathogenesis of obesity.Conclusions:These data suggest that diet, and not the obese state, was the major driving force behind gut microbiota changes. Moreover, the marked dysbiosis observed in CAF-fed rats might have resulted from the presence of several additives present in the CAF diet, or even a lack of essential vitamins and minerals. Based on our findings, we recommend the use of the prototypic WD (designed here) in DIO models. Conversely, CAF could be used to 
investigate the effects of excessive consumption of industrially produced and highly processed foods, which are characteristic of Western society.

\section{Keywords}

Animal Experiment; Animal Model; Article; Cafeteria Diet; Comorbidity; Comparative Study; Controlled Study; Diet; Diet Induced Obesity; Dysbiosis; Food Preference; Intestine Flora; Lipid Diet; Low Fat Diet; Male; Metabolic Parameters; Nonhuman; Phenotype; Priority Journal; Rat; Western Diet. 